\title{
Models for high-precision spacecraft and planetary and lunar ephemerides
}

\author{
E. Myles Standish and James G. Williams \\ JPL, California Institute of Technology, JPL 301-150, Pasadena, CA 91109, USA \\ email: ems,james.williams@jpl.nasa.gov
}

Abstract. We discuss models for high-precision spacecraft and planetary and lunar ephemerides.

Keywords. reference system, time, standards, ephemerides

The accuracies of the observational data fit by ephemerides are expected to increase by a full order of magnitude in the near future. Spacecraft ranging should improve from the present $1 \mathrm{~m}$ level to perhaps $10 \mathrm{~cm}$; directional measurements (VLBI, VLBA, etc.) will be accurate to 0.1 mas or less; and Lunar Laser Ranging measurements will be taken near the $1 \mathrm{~mm}$ level.

For such measurements to be fit by the ephemerides, a number of modeling improvements will be required for the ephemeris creation process. For the planetary ephemerides, it will be necessary to consider that many planets have distinct satellites (as opposed to being modeled with their barycenter); the perturbations of more than the just the present 300 asteroids must be considered, as well as some of the largest Kuiper belt objects; and the effect of the media through which the electromagnetic signals travel must be more accurately calibrated, possibly using dual or even triple frequency ranging.

For the lunar ephemeris, many physical and observational features must be considered and further refined: thermal expansion of the retroreflectors, a possible lunar inner core, decrease of the solar mass, refined movements of the telescopes and retroreflectors. These are in addition to the presently accounted-for features: computation of the lunar librations; nonspherical gravitational fields of the moon, earth, and sun; earth and moon tidal effects; separate modeling of the rotating lunar mantle and fluid core; atmospheric time-delays depending on pressure, temperature, and humidity at the telescope; and relativistic effects upon each station's clock, position, and light-time.

The lunar and planetary integration program is necessarily done now in quadruple precision. Relativity for the point-mass motions is complete through order $1 /\left(c^{2}\right)$; the need for the next order must be studied. Once the modeling does justice to the accuracies of the upcoming observations, a number of interesting tests will be vastly improved over the present-day status; PPN beta and gamma, $d G / d t, d A U / d t$, strong and weak equivalence principles, dark matter, etc. will be vastly improved over the present-day status: PPN beta and gamma, $d G / d t, d A U / d t$, strong and weak equivalence principles, dark matter, etc. 